\title{
Arc Spraying 3Cr13 Molten Drop Impact Stress Numerical Analysis
}

\author{
C.H. Li*, S. Wang, T.T. Zhao and Y.C. Ding \\ School of Mechanical Engineering, Qingdao Technological University, 266033 China
}

\begin{abstract}
In the thermal spraying process, the process for the molten metal particles to hit against matrix to form coating experiences great change temperature. Since the coating materials has different thermal physical properties with the matrix materials, the residual stress is surely left in the coating. Much bigger residual stress not only restricts coating thickness but also primarily affects coating binding strength. Having analyzed reason for residual stress in the thermal spraying coating and matrix, the theoretical model of arc spraying $3 \mathrm{Cr} 13$ molten drop impact stress is built and numerical simulation is done for this theoretical model. The result indicates that: the faster the molten drop speed is, the greater the pressure that matrix produces. When the molten drop's collision speed is $100 \mathrm{~m} / \mathrm{s}$, it is not obvious for the matrix's pressure stress and when the collision speed is increased to $200 \mathrm{~m} / \mathrm{s}$, the pressure stress produced in the matrix can maximize 5500Mpa; the faster the molten drop's collision speed is, the higher extent the molten drop's flattening is, which is more beneficial to increase coating's bonding strength. The radius for the molten drop in the radius of $35 \mu \mathrm{m}$ becomes $80 \sim 110 \mu \mathrm{m}$ after collision and the flat ratio of the molten drop particle is about 3 . The theoretical analysis is consistent with the experiment result.
\end{abstract}

Keywords: Arc spraying, impact stress, molten drop, coating, numerical analysis.

\section{INTRODUCTION}

During the coating process, stress is generated for deforming of the molten drop particle colliding against the matrix in a high speed or the coating for bearing particle's impact [1-7].

Particle's impact strength is proportional to kinetic energy: particle's kinetic energy can be indicated as:

$$
E_{k}=\sum_{1}^{n} \frac{1}{2} m v_{n}^{2}
$$

While the residual stress produced by collision is in linear relation with the impact strength [8]:

$$
\sigma_{\text {peening }}=K E_{k}
$$

In the formula, $\mathrm{K}$ is live load coefficient in collision.

It can be seen from Formulas (1) and (2) that, the faster the particle's speed is, the greater its kinetic energy is, thus to produce greater residual stress in the collision. However, the impact stress is generally pressure stress, which is good to counteract the residual tensile stress, which is beneficial. During the spraying process, since the molten particle collides the deposit in a high speed, stress is generated by the matrix or the formed coat deforming for bearing the impact pressure. Due to the restriction for the current equipment to test single molten drop's stress and deformation, the numerical simulation method is adopted in the paper to simulate single molten drop's deformation process and

*Address correspondence to this author at the School of Mechanical Engineering, Qingdao Technological University, 266033 China; Tel: +86532-85071757; Fax: +86-532-85071286; E-mail: sy_lichanghe@163.com analyze the influence of molten drop collision speed on residual stress and its change rule.

\section{MATHEMATICAL MODEL OF SINGLE MOLTEN DROP COLLIDING AGAINST MATRIX}

The mathematical mode for single molten drop's colliding process is based on Navier-Stokes equation and energy equation. Navier-Stokes equation is the kinematic equation to describe the momentum conservation for adhesive and incompressible fluid. The kinematic equation is the expression of momentum conservation law in fluid mechanics. Those equations can accurately describe deformation and heat conduction on molten drop's free surface [9-12].

\subsection{Fluid Dynamics Model}

This mathematical mode simulates the temporary deformation of the spherical molten drop colliding the matrix. There are several assumptions: the fluid is considered as incompressible and the fluid in the molten drop is in layer structure; the thermal property parameter of the molten drop has nothing to do with temperature; and the wetting power between the molten drop and matrix is ignored [13-15]. The flattening process of molten drop colliding the matrix is calculated by uniting and solving mass-conservation equation and momentum conservation equation. Fig. (1) is molten drop model before deformation and Fig. (2) is deforming molten drop model.

Mass-conservation equation:

$$
\frac{\partial P}{\partial \tau}+\frac{1}{M^{2}}\left(\frac{1}{R} \frac{\partial}{\partial R}\left(R U+\frac{\partial V}{\partial Z}\right)\right)=0
$$


In the formula: $\mathrm{P}$-pressure; $\tau$-time; $\mathrm{R}$-radial distance; Z-axial distance; U-radial speed; V-axial speed; $\mathrm{M}-$ mach number, $\mathrm{M}=\frac{v_{0}}{\mathrm{c}}$, c is velocity of sound.

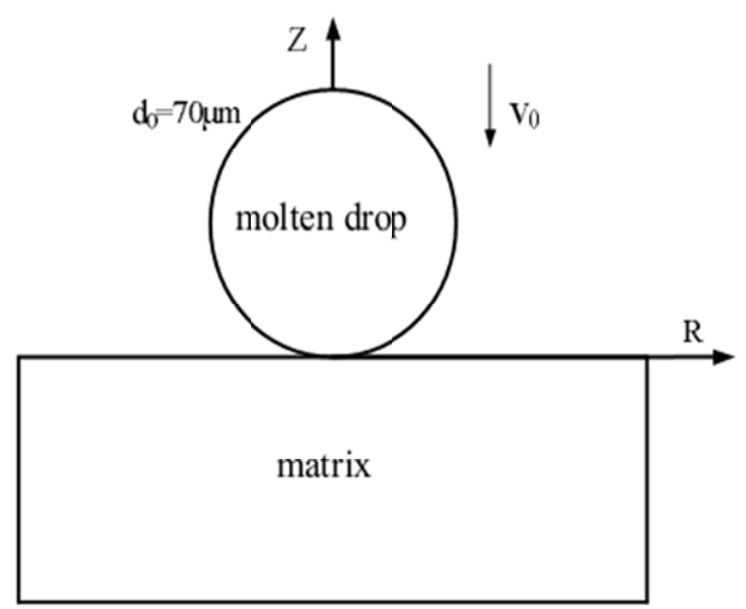

Fig. (1). Molten drop dodel before deformation.

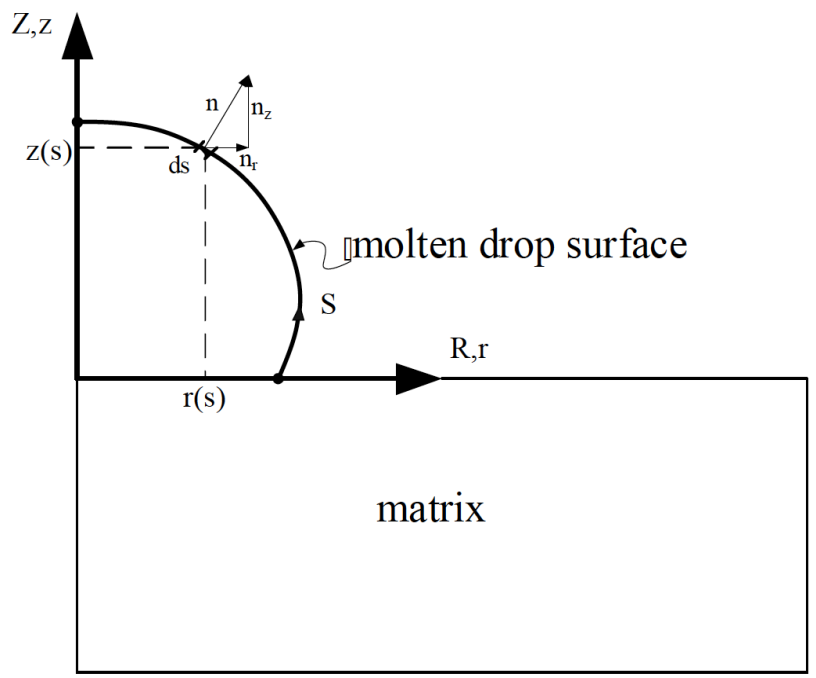

Fig. (2). Molten drop deforming model.

Momentum conservation equation in radial direction:

$\frac{\partial U}{\partial \tau}-\frac{1}{R} \frac{\partial}{\partial R}\left(R \bar{\sigma}_{R R}\right)-\frac{\partial \bar{\sigma}_{R Z}}{\partial Z}+\frac{1}{R} \bar{\sigma}_{\theta \theta}=0$

In the formula:

$\bar{\sigma}_{R R}=-P+\frac{2}{R \mathrm{e}} \frac{\partial U}{\partial R}$

$\bar{\sigma}_{\theta \theta}=-P+\frac{2}{R \mathrm{e}} \frac{U}{R}$

$\bar{\sigma}_{R Z}=\bar{\sigma}_{Z R}=\frac{1}{R \mathrm{e}}\left(\frac{\partial U}{\partial Z}+\frac{\partial V}{\partial R}\right)$
Re-Reynolds number, $\operatorname{Re}=\frac{\rho v_{0} r_{0}}{v}, \quad v$ is motion viscosity coefficient, the dimensionless number of the ratio between inertia force and viscous force.

Momentum conservation equation in axial direction:

$\frac{\partial V}{\partial \tau}-\frac{1}{R} \frac{\partial}{\partial R}\left(R \bar{\sigma}_{Z R}\right)-\frac{\partial \bar{\sigma}_{Z Z}}{\partial Z}+\frac{1}{F r}=0$

In the formula:

$\bar{\sigma}_{Z Z}=-P+\frac{2}{\operatorname{Re}} \frac{\partial V}{\partial Z}$

$\mathrm{Fr}$-Froude number, $\mathrm{Fr}=\frac{v_{0}^{2}}{\mathrm{r}_{0} \mathrm{~g}}$, the dimensionless number of the ratio between horizontal acceleration and gravitational acceleration.

\subsection{The Initial and Boundary Conditions}

Initial condition:

$\tau=0, \mathrm{U}=0, \mathrm{~V}=-1, \mathrm{P}=\frac{2}{W e}$

$W e$-Weber number, $W e=\frac{\rho r_{0} v_{0}^{2}}{v}$ is the ratio between inertia force and surface tension effect.

The boundary condition:

$\mathrm{R}=0: \mathrm{U}=0, \frac{\partial V}{\partial R}=0$

$\mathrm{Z}=0: \mathrm{U}=\mathrm{V}=0$

On the surface of the molten drop:

$\bar{\sigma}_{R R} n_{R}+\bar{\sigma}_{R Z} n_{Z}=-2 \frac{\bar{H}}{W e} n_{R}$

$\bar{\sigma}_{Z R} n_{R}+\bar{\sigma}_{Z Z} n_{Z}=-2 \frac{\bar{H}}{W e} n_{Z}$

For dimensionless treatment:

$R=\frac{r}{r_{0}}, Z=\frac{z}{r_{0}}, S=\frac{s}{r_{0}}, \bar{H}=\frac{H}{1 / r_{0}}, \tau=\frac{t}{r_{0} / v_{0}}$,

$U=\frac{\mathrm{u}}{v_{0}}, V=\frac{v}{v_{0}}, P=\frac{p}{\rho v_{0}^{2}}, \bar{\sigma}_{\mathrm{ij}}=\frac{\sigma_{\mathrm{ij}}}{\rho v_{0}^{2}}$

The average curvature of free-form surface:

$\bar{H}=\frac{r^{2}\left(r^{\prime} z^{\prime \prime}-z^{\prime} r^{\prime \prime}\right)+\left[\left(r^{\prime}\right)^{2}+\left(z^{\prime}\right)^{2}\right] r z^{\prime}}{2 r^{2}\left[\left(r^{\prime}\right)^{2}+\left(z^{\prime}\right)^{2}\right]^{3 / 2}}$

\section{THERMAL TRANSMISSION MODEL}

The temperature distribution of the molten drop and matrix is obtained by solving energy equation of the molten 
drop and matrix. The convection current and radiation on the surface in touch with the air are ignored and only the heat conduction on the contact surface between the molten drop and matrix and the heat conduction in the molten drop and matrix respectively are considered [16-19].

The dimensionless energy conservation equation of the molten drop and matrix is:

$C_{i}=\frac{\partial \vartheta_{i}}{\partial \tau}-\frac{1}{\operatorname{Pr} \operatorname{Re}}\left[\frac{1}{R} \frac{\partial}{\partial R}\left(K_{i} R \frac{\partial \theta_{i}}{\partial R}\right)+\frac{\partial}{\partial Z}\left(K_{i} \frac{\partial \theta_{i}}{\partial Z}\right)\right]=0$

In the formula:

$i=1$ represents the molten drop and $i=2$ represents matrix; $C_{i}-$ non-dimentional heat capacity; $K_{i}-$ nondimentional heat conductivity; $\mathrm{Pr}$-Prandtl number, $\operatorname{Pr}=\frac{v}{K_{i}} ; \operatorname{Re}-$ Reynolds number; $\quad \theta_{i}$-non-dimentional temperature.

$\theta_{i}=\frac{T i-\min \left(T_{1,0}, T_{2,0}\right)}{\left|T_{1,0}-T_{2,0}\right|}$

In the formula, $T_{1,0}, T_{2,0}$ represent the initial temperatures of the molten drop and matrix respectively.

\subsection{The Initial and Boundary Conditions}

Initial condition:

$\tau=0: \theta_{1}(R, Z, 0)=1, \theta_{2}(R, Z, 0)=0$

The boundary condition:

The molten drop's free surface and matrix's boundary surface:

$\frac{\partial \theta_{i}}{\partial R} n_{r}+\frac{\partial \theta_{i}}{\partial Z} n_{z}=0,(\mathrm{i}=1,2)$

Coating/matrix interface:

$\theta_{1}=\theta_{2},-k_{1} \frac{\partial \theta_{1}}{\partial Z}=-k_{2} \frac{\partial \theta_{2}}{\partial Z}$

Matrix surface far from the interface:

$\theta_{2}(R,-\infty, \tau)=\theta_{2}(\infty, Z, \tau)=0$

\section{FINITE ELEMENT OF SINGLE MOLTEN DROP COLLIDING THE MATRIX}

Since the molten drop and matrix are in axial symmetric structure, two dimension axial symmetry model is adopted in order to simplify the calculation, shown in Fig. (3). The technological experiment result indicates that, the radius of metal liquid drop in the coating is concentrated within $35 \sim 75 \mu \mathrm{m}$ that the radius of the molten drop in this paper is $35 \mu \mathrm{m}$; the matrix is a round disk with the radius of $0.5 \mathrm{~mm}$ and the height of $0.5 \mathrm{~mm}$. In fact, when the melting particles collide the matrix, the particle's speed and temperature distribution are existed in the field form. The model in this paper is simplified that it is assumed the particle's speed and temperature are well-distributed and consistent in reaching matrix and the particle's temperature and speed values on the jet flow axle replace that of the whole temperature and speed field. In addition, under different air pressure, the temperature and speed of particles in different sizes along jet flow axle are different for different spraying distances. The air pressure, molten drop temperature and speed values are shown in Table $\mathbf{1}$ at particle spraying.

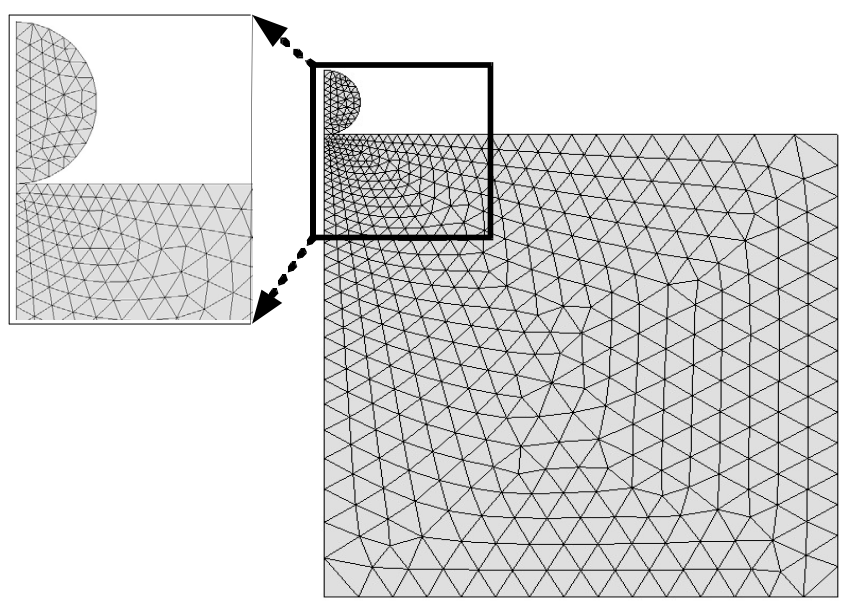

Fig. (3). Finite element model for molten drop colliding matrix process.

Table 1. Technological and Simulated Parameters

\begin{tabular}{|l|l|}
\hline Air pressure $(\mathrm{MPa})$ & 0.5 \\
\hline Molten drop temperature $(\mathrm{K})$ & 2250 \\
\hline Molten drop speed $(\mathrm{m} / \mathrm{s})$ & $100,150,200$ \\
\hline Matrix temperature $(\mathrm{K})$ & 400 \\
\hline
\end{tabular}

The coating material is $3 \mathrm{Cr} 13$ and the matrix material is ZnA115. The material's mechanical property parameters are shown in Table 2.

Table 2. Mechanical Property Parameters of $3 \mathrm{Cr} 13$ and ZnAl15

\begin{tabular}{|l|c|c|}
\hline \multicolumn{1}{|c|}{ Material } & 3Cr13 & ZnAl15 \\
\hline \hline Density $\left(\mathrm{kg} / \mathrm{m}^{3}\right)$ & 7890 & 5660 \\
\hline Elastic modulus $(\mathrm{GPa})$ & 206 & 88 \\
\hline Poisson's ratio & 0.24 & 0.2 \\
\hline Heat conductivity $(\mathrm{W} / \mathrm{m} \cdot \mathrm{k})$ & 24.9 & 113 \\
\hline Specific heat capacity $(\mathrm{J} / \mathrm{kg} \cdot \mathrm{K})$ & 460 & 450 \\
\hline Melting point $(\mathrm{K})$ & 1755 & 677 \\
\hline Thermal expansion coefficient $(1 / \mathrm{K})$ & $1.20 \mathrm{e}-5$ & $3.97 \mathrm{e}-5$ \\
\hline Latent heat of solidification $(\mathrm{J} / \mathrm{kg})$ & $3.3 \mathrm{e}+5$ & $118 \mathrm{e}+3$ \\
\hline Liquid phase temperature $(\mathrm{K})$ & 1783 & 705 \\
\hline Solid phase temperature $(\mathrm{K})$ & 1728 & 650 \\
\hline
\end{tabular}

The process of thermal spraying molten drop colliding matrix is nonlinear dynamic touch problem that the molten drop and matrix in the collision is influenced by strain, temperature and material microstructure. $\mathrm{J}-\mathrm{C}$ describes material's plastic deformation and is widely used in high 
strain rate and temperature change situations. Since thermal spraying speed is relatively low and the material is considered to be little influenced by strain rate change, therefore, J-C model after correction is adopted and the specific form is as below:

$\bar{\sigma}(\varepsilon, \hat{\theta})=\left[A+B\left(\bar{\varepsilon}^{p l}\right)^{n}\right]\left(1-\hat{\theta}^{m}\right)$

In the formula:

$\bar{\varepsilon}^{p l}$-equivalent plastic strain; $A 、 B 、 m 、 n-$ material parameters; $\hat{\theta}$-non-dimensional temperature;

$\hat{\theta}= \begin{cases}0 & \theta<\theta_{\text {transition }} \\ \frac{\left(\theta-\theta_{\text {transition }}\right)}{\left(\theta_{\text {melt }}-\theta_{\text {transition }}\right)} & \theta_{\text {transition }} \leq \theta \leq \theta_{\text {melt }} \\ 1 & \theta>\theta_{\text {melt }}\end{cases}$

$\theta_{\text {transition }}$ - material's temperature in thermal softening; Table 3 are ZnAl15 J-C parameters.

Table 3. ZnAl15 J-C Parameters

\begin{tabular}{|c|c|}
\hline J-C Parameters & ZnAl15 \\
\hline \hline $\mathrm{A}(\mathrm{MPa})$ & 170 \\
\hline $\mathrm{B}(\mathrm{MPa})$ & 410 \\
\hline $\mathrm{n}$ & 0.48 \\
\hline $\mathrm{m}$ & 0.6 \\
\hline Transition temperature $(\mathrm{K})$ & 298 \\
\hline Melting point $(\mathrm{K})$ & 677 \\
\hline
\end{tabular}

It can be seen from J-C model that, this model is only applied to the situation that material temperature is lower than its melting point temperature. When the material temperature is higher than its melting point temperature, the obtained stress is zero, which is not compliance with the actual situation. However, with the arc spraying technology, overheating may occur to the molten drop for arc effect and the molten drop's temperature reaching the matrix is higher that the melting point temperature of coating material, so J-C model can not be used as material model of the molten drop. Since the matrix temperature has always been below the melting point of matrix material during collision process, so the matrix material model adopts J-C model. For incompressible viscous fluid controlled by Navier-Stokes equation, linear Us-Up state equation is adopted to describe ABAQUS. Therefore, state equation is used to simulate volume change of the molten drop; meanwhile, Newton viscous fluid model is used to characterize deviatoric stress of the molten drop. The state equation is used to describe material's hydrodynamic feature and the relationship between the pressure bore by fluid and the current volume can be obtained by uniting and solving energy equation and state equation in explicit solution.

According to energy conservation equation, the internal energy increased by each unit mass is equal to the sum of stress power and heat's increasing rate. The energy equation ignoring heat conduction is as follow:

$\rho \frac{\partial E_{m}}{\partial t}=\left(p-p_{b v}\right) \frac{1}{\rho} \frac{\partial \rho}{\partial t}+S: \dot{e}+\rho \dot{Q}$

In the formula:

$p$-pressure stress. It is positive when it is pressure stress;

$p_{b v}$ - the pressure stress produced for volume viscosity;

$S$-deviatoric stress tensor;

$\dot{e}$-deviatoric strain rate;

$\dot{Q}$ - heat consumption rate in unit mass.

According to state equation, the pressure is the function of the current material density and unit mass's internal energy. It defines material's balance state, just as the formula below:

$p=f\left(\rho, E_{m}\right)$

The relationship between pressure stress $p$ and the current volume $V$ can be obtained by uniting Formulas 5 and 6 to remove internal energy and this relationship is unique called Hugoniot curve. Hugoniot pressure is density function, generally obtained by experiment data. The general form of state equation is:

$p=f+g E_{m}$

$f$ and $g$ in the formula are only density function and their value depends on specific state equation. State equation in multiple forms is provided in ABAQUS and Mie-Gruneisen state equation is adopted in this paper to simulate volume strength of the molten drop.

Mie-Gruneisen state equation is linear in energy. The general form is:

$p-p_{H}=\Gamma \rho\left(E_{m}-E_{H}\right)$

In the formula:

$\Gamma=\Gamma_{0} \frac{\rho_{0}}{\rho}$

$E_{H}=\frac{p_{H} \eta}{2 \rho_{0}}$

where, $p_{H}$-Hugoniot pressure; $E_{H}$ - specific energy per unit mass, it is only the density function; $\Gamma$ - Gruneisen coefficient; $\Gamma_{0}$-material constant; $\rho_{0}$-reference density; $\eta=1-\rho_{0} / \rho$, nominal volume compression strain.

It is obtained by uniting Formulas (28)-(30) to remove $\Gamma$ and $E_{H}$ :

$p=p_{H}\left(1-\frac{\Gamma_{0} \eta}{2}\right)+\Gamma_{0} \rho_{0} E_{m}$

In the formula: 
$p_{H}=\frac{\rho_{0} c_{0}^{2} \eta}{(1-s \eta)^{2}}$

$U_{s}=c_{0}+s U_{p}$

$U_{s}$-shock wave rate; and $U_{p}$-molten drop rate;

It is obtained by uniting and solving Formulas (31)-(33):

$p=\frac{\rho_{0} c_{0}^{2} \eta}{(1-s \eta)^{2}}\left(1-\frac{\Gamma_{0} \eta}{2}\right)+\Gamma_{0} \rho_{0} E_{m}$

Mie-Gruneisen state equation can be fixed only when four parameters of $c_{0}, s, \Gamma_{0}$ and $\rho_{0}$ are confirmed.

3Cr13's Mie-Gruneisen state equation parameters are given in Table 4.

Table 4. 3Cr13's Mie-Gruneisen State Equation Parameters

\begin{tabular}{|c|c|c|c|}
\hline$\rho_{\mathbf{0}}\left(\mathrm{kg} / \mathbf{m}^{3}\right)$ & $c_{\mathbf{0}}(\mathbf{m} / \mathbf{s})$ & $s$ & $\Gamma_{\mathbf{0}}$ \\
\hline \hline 7890 & 4610 & 1.73 & 1.67 \\
\hline
\end{tabular}

It is known from elasticity mechanics that stress tensor can be broken into spherical tensor and deviator. The state equation describes material's volume change; and also characterizes spherical tensor. The deviator is also considered and Newton viscous model is used to characterize deviator.

For viscous body, deviatoric stress is related to deviatoric strain rate:

$S=2 \eta \dot{e}=\eta \dot{\gamma}(35)$

In the formula:

$\mathrm{S}-$ deviatoric stress; $\dot{e}-$ deviatoric strain rate; $\eta-$ viscidity; $\dot{\gamma}$-project shear strain rate, $\dot{\gamma}=2 \dot{e}$.

The change values of $3 \mathrm{Cr} 13$ viscosity along with temperature are given in Table $\mathbf{5}$.

Table 5. The Change Values of $3 \mathrm{Cr} 13$ Viscosity Along with Temperature

\begin{tabular}{|c|c|}
\hline $\mathbf{T}(\mathbf{K})$ & $\eta(\mathbf{P a} \cdot \mathbf{S})$ \\
\hline \hline 500 & 0.01 \\
\hline 1000 & 0.008 \\
\hline 2300 & 0.0025 \\
\hline
\end{tabular}

The molten drop is deformed in colliding the matrix and expanded into the coating. If the molten drop does not separate from the matrix after colliding the matrix, for the contact among surfaces, ABAQUS/Explicit defaults to use dynamics contact formula that it applies forecast/correction method to get the accurate flexibility under the contact condition. It is assumed no contact at the beginning of the increment step, if interference is occurred at the end of increment step, the acceleration value is corrected to get correct configuration to enhance contact constraint. Since the involved question is the contact between the molten drop surface and matrix surface, the dynamics contact formula is adopted. Moreover, the friction coefficient on the metal surface is generally 0.1 , so it is assumed to set the friction coefficient on the molten drop surface and matrix surface is 0.1 .

The dynamic display temperature-displacement coupling analysis method is adopted for analysis and ABAQUS/Explicit provides 11 units for the analysis. CAX3T unit is adopted since it is the coupling unit of threenode axisymmetric linear displacement and temperature. The size is several micron grains and the flattening process is completed in $1 \mu$ s or less than $1 \mu$ s order of magnitude and the analysis time is $2 \mu \mathrm{s}$. The degree of freedom at the matrix bottom is fixed as well as symmetry axis and axial symmetry boundary condition.

\section{RESULT AND ANALYSIS}

The molten drop impact stress distribution for matrix at different speed is given in Fig. (4). The molten drop's deformation is shown in Fig. (5). It can be seen from Figs. $(4,5)$ that, the faster the molten drop's speed is, the flattening is more complete and the bigger pressure stress the matrix produces. The produced pressure stress is good to coating and matrix that it can counteract partial hot drawing stress. The bigger the pressure stress is, the more the counteracted tensile stress is. If the final stress status of the coating is pressure stress, it will be good for coating formation.

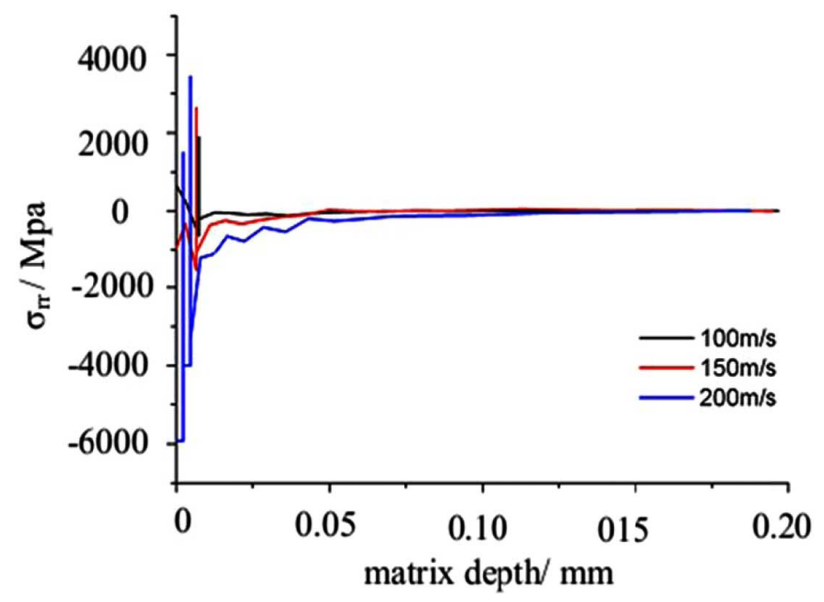

Fig. (4). $\sigma_{\mathrm{rr}}$ distribution in matrix thickness direction after $2 \mu \mathrm{s}$.

The molten drop's radius change under different collision speeds is given in Fig. (6). It is known from the figure that at the beginning of the collision, the faster the molten drop's speed is, the bigger its deformation rate is; the molten drop's radius maintains unchanged after about $1 \mu \mathrm{s}$. The radius of the molten drop in the radius of $35 \mu \mathrm{m}$ becomes $80-110$ after collision. The flatness ratio of the molten drop particle (the ratio of disk diameter and the initial particle diameter after particle's deformation) is about 3 , which is compliance with the research result in the technology experiment: under the thermal spraying condition, the metal particle's flatness ratio is generally 3-5.

Considering the heat conduction in the molten drop, the matrix and the interface between the molten drop and the 


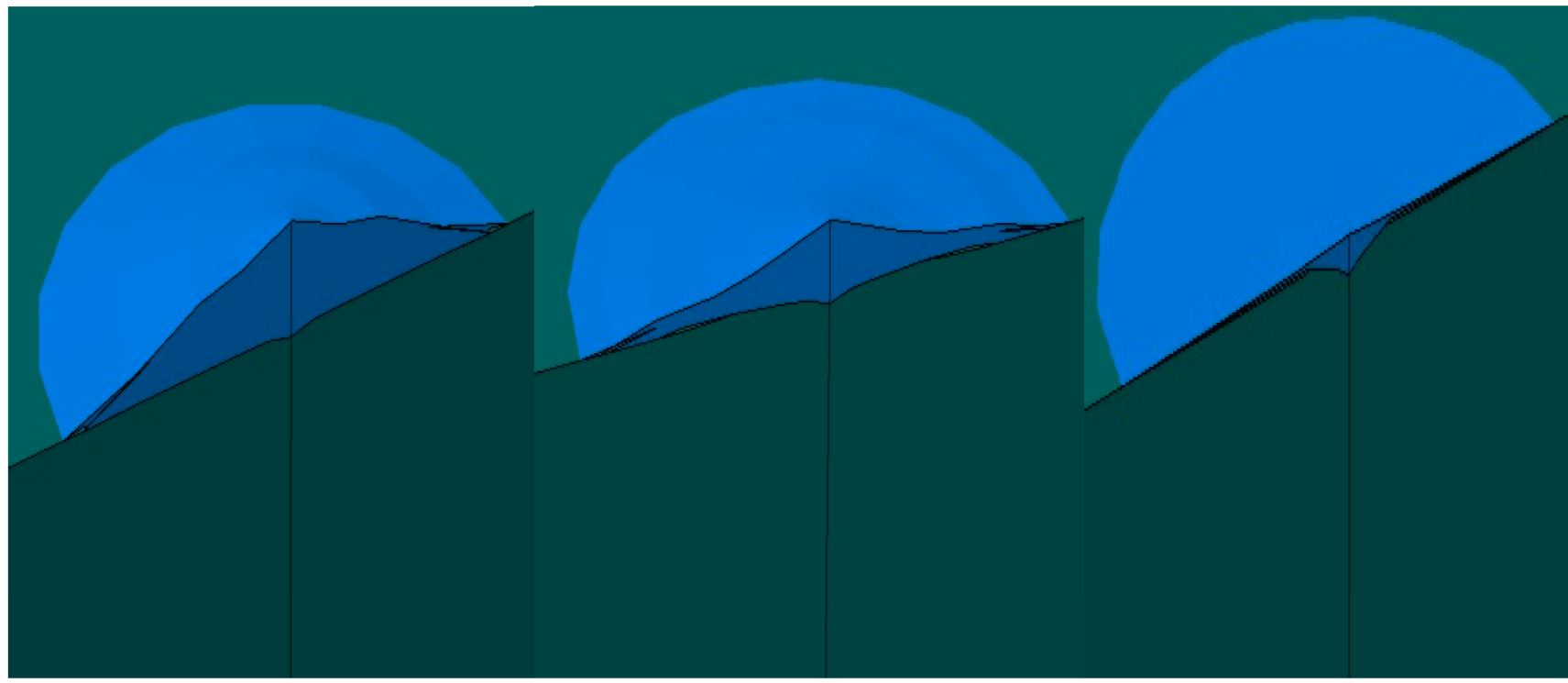

( a ) $\mathrm{v}=100 \mathrm{~m} / \mathrm{s}$;

(b ) $v=150 \mathrm{~m} / \mathrm{s}$;

(c ) $v=200 \mathrm{~m} / \mathrm{s}$

Fig. (5). Matrix deformation result under molten drop's impact at different speeds.

matrix, the temperature change of matrix and the molten drop for the molten drop at different speeds after collision of is given in Fig. (7). It can be seen from the figure that, the molten drop increases $20 \mathrm{~K}$ temperature; and also because the molten drop passes the heat to the matrix, so there is about $10 \mathrm{~K}$ temperature rise for matrix in the scope of $33 \mu \mathrm{m}$. The faster the speed is, the higher the temperature rise is. Since $90 \%$ kinetic energy is transferred into heat during the molten drop's collision process, so, there is temperature rise for the molten drop.

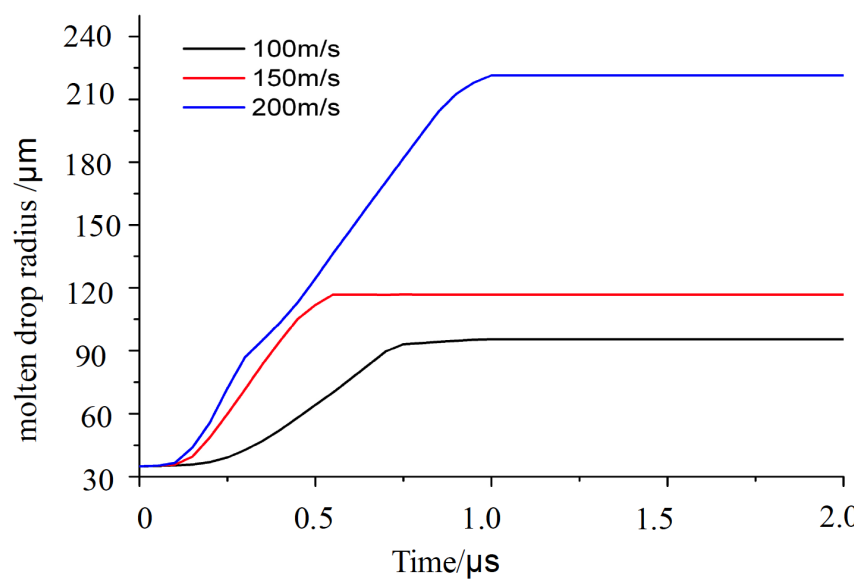

Fig. (6). Radius change during molten drop's collision process.

\section{CONCLUSION}

The molten drop's collision speed has significant influence on the produced impact stress. The faster the spraying speed is, the bigger the pressure stress produced in the matrix. It can be seen from the result that when the molten drop's collision speed is $100 \mathrm{~m} / \mathrm{s}$, the matrix's pressure stress is not obvious; when the collision speed is

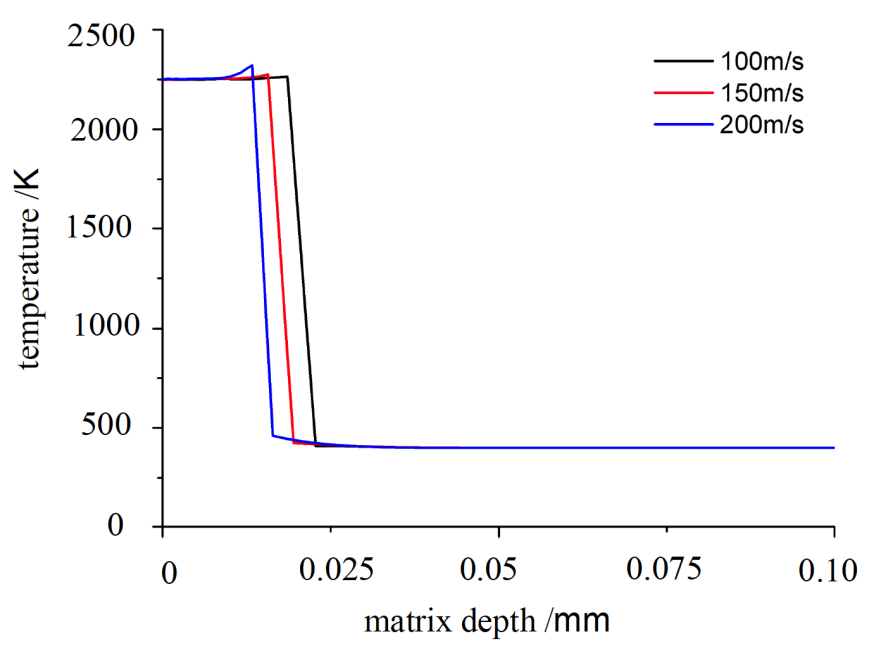

Fig. (7). Temperature distribution of the molten drop and matrix in the thickness direction after collision.

increased to $200 \mathrm{~m} / \mathrm{s}$, the pressure stress produced in the matrix can maximize $5500 \mathrm{MPa}$ at its maximum. It is also visually seen from the result that, the faster the molten drop's collision speed is, the higher the flattening extent of the molten drop is, thus it is better for coating bond strength improvement. In addition, since most collision kinetic energy of the molten drop is transferred into heat, so, the temperature of the molten drop and matrix also has increased along with molten drop's different collision speeds. It can be seen from the result that the faster the speed is, the greater the temperature increase is.

Molten drop's spraying speed needs to increase to generate greater pressure stress to counteract the tensile stress produced by temperature difference. The temperature difference between coating and matrix is reduced by decreasing coating temperature or increasing matrix 
temperature thus to reduce coating's hot drawing stress, increase coating thickness and improve coating property.

\section{CONFLICT OF INTEREST}

The authors confirm that this article content has no conflicts of interest.

\section{ACKNOWLEDGEMENTS}

This research was financially supported by the National Natural Science Foundation of China (50875138; 51175276); the Shandong Provincial Natural Science Foundation of China (ZR2009FZ007); Qingdao science and technology program of basic research projects (12-1-4-4(1)-jch) and the Specialized Construct Fund for Taishan Scholars.

\section{REFERENCES}

[1] Bansal P, Shipway PH, Leen SB. Residual stresses in high-velocity oxy-fuel thermally sprayed coatings-modelling the effect of particle velocity and temperature during the spraying process. Acta Mater 2007; 55(15): 5089-101.

[2] Pasandideh-Fard M, Chandra S, Mostaghimi J. A threedimensional model of droplet impact and solidification. Int J Heat Mass Transfer 2002; 45(11): 2229-42.

[3] Waldvogelt JM, Poulikakos D. Solidification phenomena in picoliter size solder droplet deposition on a composite substrate. Int J Heat Mass Transfer 1997; 40(2): 295-309.

[4] Rajneesh B, Jon P. Longtin, Attinger D. A numerical investigation on the influence of liquid properties and interfacial heat transfer during microdroplet deposition onto a glass substrate. Int J Heat Mass Transfer 2007; 50(15/16): 2912-23.

[5] Zhenghao G, Heong Wah NG, Devasenapathi A. Depositioninduced residual stresses in plasma-sprayed coatings. Surface Coatings Technol 2004; 187(2/3): 307-19.

[6] Li CH, Hou YL, Liu ZR, Ding YC. Investigation into temperature field of nano-zirconia ceramics precision grinding. Int J Abrasive Technol 2011; 4(1): 77-89.
[7] Gan Z, Heong Wah NG, Devasenapathi A. Deposition-induced residual stresses in plasma-sprayed coatings. Surface Coatings Technol 2004; 187(2/3): 307-19.

[8] Yongxiong C, Xiubing L, Yan L, Jinyuan B, Binshi X. Finite element modeling of coating formation and transient heat transfer in the electric arc spray process. Int J Heat Mass Transfer 2010; 53(9/10): 2012-21

[9] Li CH, Hou YL, Ding YC, Cai GQ. Feasibility investigations on compound process: a novel fabrication method for finishing with grinding wheel as restraint. Int J Comput Mater Sci Surf Eng 2011; 4(1): 55-68.

[10] Tsui YC, Clyne TW. An analytical model for predicting residual stresses in progressively deposited coatings-part I:planar geometry. Thin Solid Films 1997; 306(1): 23-33.

[11] Li CH, Han ZL, Du C, Ding YC. Numerical study on critical speed modeling of ultra-high speed grinder spindle. Comm Comp Inform Sci 2011; 201(1): 202-9

[12] Li CH, Wang S, Ding YC. The theoretical and experimental investigation into technological parameters of high-melting metal arc spraying. Open Mater Sci J 2012; 6: 60-7.

[13] Rayment T, Hoile S, Grant PS. Phase transformations and control of residual stresses in thick spray-formed steel shells. Metall Mater Trans 2004; 35(6): 1113-22.

[14] He ZY, Lu BH. Key technologies and application of metal arc spray tooling. J Plast Eng 2008; 15(2): 65-9.

[15] Karunakaran KP, Shanmuganathan PV, Jadhav SJ. Rapid prototyping of metallic parts and moulds. J Mater Process Technol 2000; 105(3): 371-81.

[16] Li CH, Liu ZR, Liu GY, Ding YC. Experimental investigations of mechanical characteristics and tribological mechanisms of nanometric zirconia dental ceramics. Open Mater Sci J 2011; 5: 178-83.

[17] Li CH, Hou YL, Du C, Ding YC. An analysis of the electric spindle's dynamic characteristics of high speed grinder. J Adv Manuf Syst 2011; 10(1): 159-66.

[18] Gideon NL, Schindel R, Kruth JP. Rapid manufacturing and rapid tooling with layer manufacturing $(\mathrm{lm})$ technologies, state of the art and future prespectives. Ann CIRP 2010; 47(2): 651-71.

[19] Hou YL, Li CH, Han ZL, et al. Examination of the material removal mechanisms during the abrasive jet finishing of 45 steel. Adv Sci Lett 2011; 4(4-5): 1478-1484. 